\title{
New superatom in alkali-metal atoms
}

\author{
Sergey I. Pokutnyi · Petr P. Gorbyk
}

Received: 28 June 2014/ Accepted: 14 September 2014/Published online: 30 September 2014

(c) The Author(s) 2014. This article is published with open access at Springerlink.com

\begin{abstract}
On the basis of a similarity with electronic states in alkali-metal atoms, the existence of a new artificial atom (superatom) has been predicted theoretically. This superatom may be considered as a new alkali-metal atom.
\end{abstract}

Keywords Superatoms - Artificial atoms - Quasi-zerodimensional nanostructures · Quantum dots · Alkali-metal atom $\cdot$ Separated electrons and holes

\section{Introduction}

The concept of superatom (or artificial atom) was very fruitful for the development of mesoscopic physics and chemistry [1-4]. As an example of superatom, one can consider a quantum dot (QD). Such an analogy is justified by the similarity of their spectra of discrete electronic states [1-4].

In our works $[1,2,4]$, we have proposed a new model of superatom in the form of a quasi-zero-dimensional heterogeneous nanosystem. Such a nanosystem is a spherical QD (the superatom nucleus) of radius $a$ consisted of semiconductor with permittivity $\varepsilon_{2}$, and surrounded with a dielectric matrix with permittivity $\varepsilon_{1}$. A hole $h$ with effective mass $m_{h}$ can move around the bulk of QD and an electron $e$ with effective mass $m_{e}^{(1)}$ is located in the dielectric matrix. Beginning from the QD critical size of $a \geq a_{c}^{(1}$ (about $4 \mathrm{~nm}$ ), the superatom energy spectrum consists of quantum-sized discrete levels only. Let us call

S. I. Pokutnyi ( $\square)$ ? P. P. Gorbyk

Chuiko Institute of Surface Chemistry, National Academy of

Sciences of Ukraine, 17 General Naumov Str., Kiev 03164,

Ukraine

e-mail: pokytniy@mail.ua; pokutnyi_sergey@inbox.ru this kind of superatom the hydrogen-like superatom [1, 2, 4]. In such a superatom, an electron localized above the QD surface is a valence one. The quantum-sized discrete energy levels of the superatom are located inside the band gap of the dielectric matrix. The superatom electrons are bound on the well-defined orbitals and localized in the vicinity of the superatom nucleus (QD) $[1,2,4]$.

The artificial atoms have the ability to add $N$ electrons to their electron orbitals, where $N$ can vary from one to several tens and even be more than the serial numbers for all known elements in the periodic table [1, 2, 4]. This new property of the attachment of $N$ electrons to the electronic orbitals in the artificial atoms leads to their high reactivity and new practical applications due to their strong oxidative activity, possibility increase substantially the intensity of photochemical reactions in the processes of adsorption and catalysis, as well as their ability to form many new compounds with unique properties (in particular, quasi-molecules and quasi-crystals) [4-6]. Therefore, the theoretical studies aimed at prediction of new artificial atoms (not presented in the periodic table) and possibility of their existence as well as the experimental studies of their properties are very relevant.

Quantum discrete states of an isolated alkali-metal atom are determined by the motion of a single outmost valence electron around a symmetric atomic core (the nucleus and remaining electrons) [7]. The energy spectra of the hydrogen-like superatom are formed by the quantum-sized discrete energy levels of the valence electron [1, 2, 4]. Thus, the similarity between the spectra of discrete electronic states of superatoms and ones of isolated alkalimetal atoms as well as the similarity of their chemical activity are observed [1, 2, 4-7].

In this article, we have theoretically predicted a new artificial alkali-metal-like atom using the electronic state 


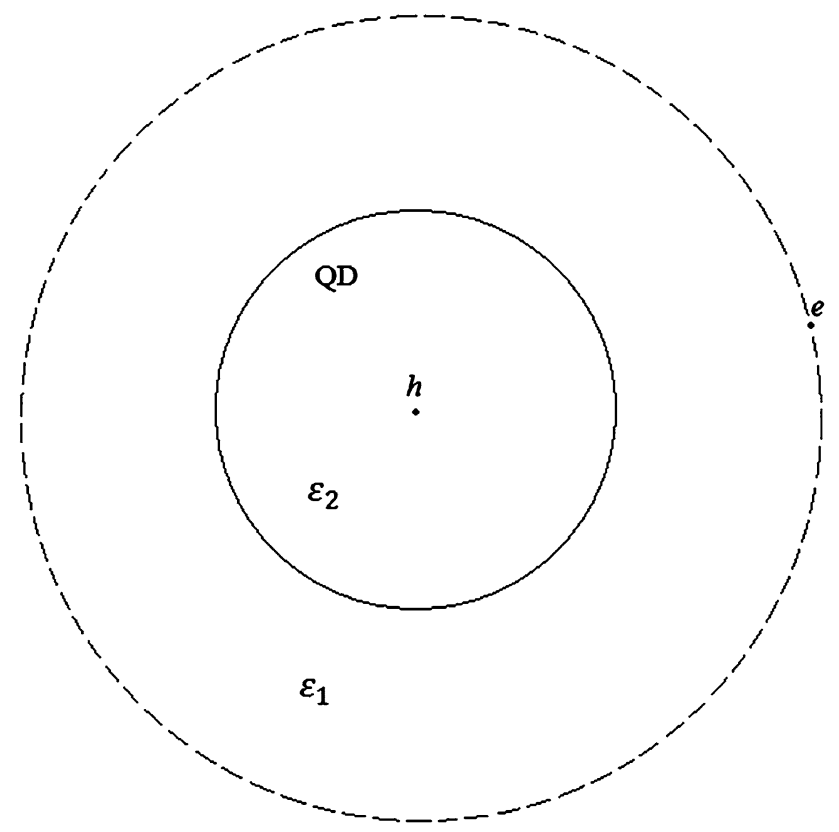

Fig. 1 New model of superatom, which is quasi-zero-dimensional nanosystem consisting of a spherical QD (the superatom nucleus with a radius of $a$ ) of semiconductor (dielectric) with the dielectric constant $\varepsilon_{2}$ surrounded by a dielectric matrix with the dielectric constant $\varepsilon_{1}$. The hole $h$ with the effective mass $m_{h}$ moves in the QD bulk, while the electron $e$ with the effective mass $m_{e}^{(1)}$ is located in the dielectric matrix

spectroscopy analogy between an artificial atom and an isolated alkali-metal atom.

\section{The new model of an artificial atom}

In the works [1, 2, 4], a new model of superatom has been proposed. According to the model, the superatom is a quasi-zero-dimensional heterogeneous nanosystem consisting of a spherical QD (the superatom nucleus of a radius a) whose bulk is semiconductor (dielectric) with a dielectric constant $\varepsilon_{2}$, surrounded by a dielectric matrix with a dielectric constant $\varepsilon_{1}$ (with a relative dielectric constant $\left.\varepsilon=\left(\varepsilon_{2} / \varepsilon_{1}\right) \gg 1\right)$ (see Fig. 1). A hole $h$ with the effective mass $m_{h}$ moves in the QD bulk, while an electron $e$ with the effective mass $m_{e}^{(1)}$ is contained in the dielectric matrix. In such a heterogeneous nanostructure, the lowest electronic level is located in the matrix and the lowest hole level is in the QD bulk. The large shift of the valence band (about $700 \mathrm{meV}$ ) results in localization of holes in the QD bulk. The large shift of the conduction band (about $400 \mathrm{meV}$ ) creates a potential barrier for electrons (the electrons move in the matrix and do not penetrate into the QD bulk) $[1,2,4]$. The energy of Coulomb interaction between the electron and the hole, and the energy of polarization interaction between the electron and the QD- matrix interface (since the QD dielectric constant $\varepsilon_{2}$ is much more than the matrix dielectric constant $\varepsilon_{1}$ ) produce localization of the electron in the potential well above the QD surface [1,2].

When the QD radius $a$ increases and becomes $a>>a_{\mathrm{ex}}^{0}$, where

$a_{\mathrm{ex}}^{0}=\frac{2 \varepsilon_{1} \varepsilon_{2}}{\varepsilon_{1}+\varepsilon_{2}} \cdot \frac{\hbar^{2}}{\mu_{0} e^{2}}$

is the two-dimensional Bohr electron radius $[e$ is the electron charge, $\mu_{0}=m_{e}^{(1)} m_{h} /\left(m_{e}^{(1)}+m_{h}\right.$ the reduced mass of the electron-hole pair (spatially separated electron and hole)], the QD-matrix spherical interface becomes flat. In this artificial atom, the electron localized above the QDmatrix interface becomes two-dimensional.

At that, the main term of the potential energy in the Hamiltonian function describing the motion of electron in the superatom is the energy of Coulomb electron-hole interaction $[1,2]$ :

$V_{\mathrm{eh}}(r)=-\frac{1}{2}\left(\frac{1}{\varepsilon_{1}}+\frac{1}{\varepsilon_{2}}\right) \frac{e^{2}}{r}$,

(where $r$ is a distance from the electron to the QD center). When the QD-matrix interface is spherical, the energy of polarization interaction between the electron and the hole makes a significantly lesser contribution to the potential energy of the Hamiltonian. As a first approximation, such a contribution may be neglected $[1,2]$. In this case, the twodimensional electron energy spectrum in the artificial atom becomes as follows $[1,2]$ :

$E_{n}=-\frac{R y_{\mathrm{ex}}^{0}}{(n+(1 / 2))^{2}}, R y_{\mathrm{ex}}^{0}=\frac{\left(\varepsilon_{1}+\varepsilon_{2}\right)^{2}}{4 \varepsilon_{1}^{2} \varepsilon_{2}^{2}} \cdot\left(\frac{\mu_{0}}{m_{0}}\right) \mathrm{Ry}_{0}$,

where $n=0,1,2$ is the principal quantum number of the electron, $R y_{0}=13.606 \mathrm{eV}$ the Rydberg constant. The binding energy of the ground state of the two-dimensional electron, according to (3), is given by:

$E_{\mathrm{ex}}^{0}=-4 R y_{\mathrm{ex}}^{0}$.

The electron binding energy in the ground state (4) means the energy required for decay of the electron-hole bound state $(n=0)$.

The dependence of the electron binding energy $E_{\mathrm{ex}}(a, \varepsilon)$ derived in $[1,2]$ by the variational method for the ground state of the superatom consisting of the zinc selenide QD with the radius $a$ in the borosilicate glass matrix [8] shows that the electron-bound state arises in the vicinity of the QD-matrix spherical interface starting with the critical QD radius value of $a \geq a_{c}{ }^{(1)}=3.84 \mathrm{~nm}$. At that, the hole is in motion inside the QD bulk and the electron is localized above the QD-matrix spherical interface. In this case, the energy of Coulomb interaction $V_{\mathrm{eh}}(r)$ (2) between the 
electron and the hole and also the energy of polarization interaction between the electrons or the hole and the QDmatrix spherical interface exceed the energy of electron or hole size quantization in the artificial atom. Thus, the works $[1,2]$ have shown that the process of superatom formation has a threshold nature and the superatom will be formed only starting with the QD radius of $a \geq a_{c}{ }^{(1)}=$ $3.84 \mathrm{~nm}$.

When the QD radius $a$ increases, the electron binding energy for the superatom ground state also increases. In the range of radii $4.0 \leq a \leq 29.8 \mathrm{~nm}$, the electron binding energy for the superatom ground state significantly exceeds (by a factor of 4.1-76.2) the value of the exciton binding energy $\tilde{E}_{\mathrm{ex}}^{0} \approx 21.07 \mathrm{meV}$ in a single crystal of zinc selenide [1, 2]. Starting with the QD radius $a$ ge $a_{c}{ }^{(2)}=29.8$ $\mathrm{nm}$, the electron binding energy for the superatom ground state tends asymptotically to the value $E_{\mathrm{ex}}^{O}=-1.5296 \mathrm{eV}$, which characterizes the binding energy of the two-dimensional electron in the ground state of the artificial atom (4) $[1,2]$.

The significant increase in the electron binding energy of the superatom ground state is mainly determined by two factors [1, 2]: (1) a significant increase in the energy of electron-hole Coulomb interaction $\left|V_{\mathrm{eh}}(r)\right|(2)$ (the effect of "dielectric enhancement" [9]);2) the spatial restrictions imposed on the region of quantification by the QD volume; at that, upon increasing the QD radius $a$ from the value of a ge $a_{c}^{(2)}=52 a_{\mathrm{ex}}^{0}=29.8 \mathrm{~nm}$, the superatom becomes twodimensional with the ground state binding energy $E_{\mathrm{ex}}^{0}(4)$, whose value exceeds the exciton binding energy in a single crystal of zinc selenide by a factor of 100 . The effect of "dielectric enhancement" is due to an electron-hole interaction within the superatom and a significant role played by the electric field created by quantum dots in the matrix in this interaction when the dielectric constant $\varepsilon_{1}$ of the matrix is much less than the dielectric constant $\varepsilon_{2}$ of the QDs. At that, the interaction between the electron and the hole in the superatom is significantly larger than in the semiconductor with a dielectric constant of $\varepsilon_{2}$ [9].

\section{New artificial atom, which is similar to a new single alkali-metal atom}

Quantum discrete states of the individual atoms of alkali metals are determined by the movement of only one, the outermost valence electron around a symmetric atomic core (containing the nucleus and the remaining electrons) [7]. At large distance $r$ between the electron and the nucleus (so that $r \gg a_{0}$, where $a_{0}=0.053 \mathrm{~nm}$ is the Bohr radius of the electron in the hydrogen atom), the field of the atomic core is described by the Coulomb field [7]:
$\mathrm{V}(r)=-\left(Z e^{2} / r\right)$,

determining the interaction of the valence electron with the atomic core ( $Z$ is the atom serial number in the periodic table). The energy spectrum of a single alkali-metal atom is described by the hydrogen-like spectrum [7]:

$E_{n *}=-\frac{R y^{*}}{\left(n^{*}\right) 2}, \quad R y *=Z^{2} R y_{0}$,

where $n *=(n+y)$ is the effective quantum number ( $n=1,2,3, \ldots$ is the principal quantum number), the correction $y$ depends on the orbital quantum number $l$. The correction $y$ is due to the fact that the valence electron moves in the Coulomb field of the atomic core, where the nuclear charge is screened by core electrons. The correction $y$ is determined by comparing spectrum (6) with the experimental values. The correction $y$ is negative $(y<0)$, and the more close is the valence electron orbit to the atomic core the larger is the numerical value of $y$. The number of possible orbits along which the valence electron can move in a single alkali-metal atom is the same as in the hydrogen-like atom [7].

The similarity of the individual series of neutral alkalimetal atoms with the Balmer series of the hydrogen atom allows to suggest that the energy spectra of neutral alkalimetal atoms are produced by transitions of the valence electron from higher levels to the level with the principal quantum number $n=2$ [7].

In a single alkali-metal atom, the valence electron is moving in the Coulomb field of the atomic core (5) with the same functional dependence on $r$ as the Coulomb field (2), in which the valence electron of the hydrogen-like artificial atom is moving. This fact allows us to describe the energy spectra of the valence electrons in a single alkali-metal atom (6) and in the artificial atom (3) with the use of a hydrogen-like spectrum At the same time, the number of possible quantum-dimensional states of the valence electron in the model of hydrogen-like artificial atom is the same as the number of quantum discrete states of the valence electron in a single alkali-metal atom $[1,2,4,7]$.

Table 1 Position of energy levels of the valence electron in some alkali-metal atoms $(K, \mathrm{Rb}, \mathrm{Sc})$ and a new artificial atom $\mathrm{X}$. Level shifts of the valence electron $\left(\Delta E_{x}^{\mathrm{Sc}}, \Delta E_{x}^{\mathrm{Sc}}, \Delta E_{\mathrm{Sc}}^{\mathrm{Rb}}\right)$ relative to the adjacent level

\begin{tabular}{lll}
\hline $\begin{array}{l}\text { Alkali-metal } \\
\text { atoms selected }\end{array}$ & $\begin{array}{l}\text { Valence electron } \\
\text { energy levels }(\mathrm{meV})\end{array}$ & $\begin{array}{l}\text { Level shifts of the } \\
\text { valence electron }(\mathrm{meV})\end{array}$ \\
\hline $\mathrm{K}$ & -721.1 & \\
$\mathrm{Rb}$ & -711.2 & 10 \\
$\mathrm{Sc}$ & -652 & 59 \\
$\mathrm{X}$ & -593 & 59 \\
\hline
\end{tabular}


Table 1 shows the positions of the valence electron energy levels in the individual atoms of alkali metals $(K$, $\mathrm{Rb}, \mathrm{Sc}$ ) [7] and the new artificial atom $X$, as well as the shifts of the valence electron levels $\left(\Delta E_{\mathrm{Rb}}^{k}, \Delta E_{\mathrm{Sc}}^{\mathrm{Rb}}, \Delta E_{x}^{\mathrm{Sc}}\right)$ relative to the adjacent levels.

Let us assume that the shift of the energy level $E_{x}$ in the artificial atom $X$ (relative to the energy level $E_{\mathrm{sc}}$ in the Sc atom) will be the same as the shift of the energy level $E_{\mathrm{Rb}}$ in the $\mathrm{Rb}$ atom (relative to the energy level $E_{\mathrm{sc}}$ in the $\mathrm{Sc}$ atom), (i.e., $\left(\Delta E_{x}^{\mathrm{Sc}}=\Delta E_{\mathrm{Sc}}^{\mathrm{Rb}}\right)$. Then, the valence electron level in the artificial atom will be $E_{x}=-593 \mathrm{meV}$. In the works $[1,2]$ with the use of the variation method, we derived the relationship for the electron binding energy $E_{\mathrm{ex}}(a, \varepsilon)$ of the ground state in the artificial atom consisted of the $\mathrm{ZnSe}$ quantum dot in the borosilicate glass matrix. From this relationship, we can find the radius of the zinc selenide quantum dot $a_{1}=5,4 \mathrm{~nm}$, which corresponds to the level $E_{x}=-593 \mathrm{meV}$. It should be noted that the energy levels of the valence electron in the individual alkali-metal atoms $(K, \mathrm{Rb}, \mathrm{Sc})$ [7] and the new artificial atom $\mathrm{X}$ are located in the infrared region.

\section{Conclusions}

In summary, we have proposed a new model of an artificial atom in the form of a quasi-atomic heterostructure consisted of the spherical QD (the superatom nucleus of zinc selenide) with a radius of $a$ in the surrounding matrix of borosilicate glass (the hole $h$ with the effective mass $m_{h}$, is moving in the QD bulk and the electron with the effective mass $m_{b}^{(1)}$ is located in the matrix). The model allows us to find a new artificial atom $X$ (not presented in the periodic table) that is a new analogue of isolated alkali-metal atoms. In this artificial atom, the valence electron can participate in various physical $[1-6,8,10]$ and chemical $[4,6]$ processes in much the same way as the atomic valence electrons can do in various atomic systems (in particular, in isolated alkali-metal atoms [7] ). These processes are possible because of new unique properties of such artificial atoms: strong oxidizing ability, potentialities for increasing the rates of photochemical reactions during catalysis and adsorption as well as the ability to form a great number of new compounds with unique characteristics (in particular, quasi-molecules and quasi-crystals [4-6]).
Small exciton binding energies in the QDs are the main factor that hampers the use of semiconductor nanoheterostructures as active regions of nanolasers [1-3]. Therefore, the researches into nanoheterostructures with significantly increased binding energies of local electronic states in quantum dots are of current importance [1-3]. The effect of significantly increased electron binding energies in the hydrogen-like superatom [1,2] can be used for experimental researches into the existence of such superatoms at room temperatures and will stimulate experiments with nanoheterostructures on the basis of superatoms as the effective active region for nanolasers operated on optical transitions.

Conflict of interest The authors declare that they have no competing interests.

Open Access This article is distributed under the terms of the Creative Commons Attribution License which permits any use, distribution, and reproduction in any medium, provided the original author(s) and the source are credited.

\section{References}

1. Pokutnyi, S.I.: Binding energy of the exciton of a spatially separated electron and hole in quasi-zero-dimensional semiconductor nanosystems. Tech. Phys. Lett. 39, 233 (2013)

2. Pokutnyi, S.I.: On an exciton with a spatially separated electron and hole in quasi-zero-dimensional semiconductor nanosystems. Semiconductors 47, 791 (2013)

3. Pokutnyi, S.I.: Exciton states in semiconductor quantum dots in the framework of the modified effective mass method. Semiconductors 41, 1323 (2007)

4. Pokutnyi, S.I., Gorbyk, P.P.: Superatoms in quasi-zero-dimensional nanostructures. J. Appl. Chem. 1, 44 (2013)

5. Pokutnyi, S.I.: Biexcitons formed from spatially separated electrons and holes in quasi-zero-dimensional semiconductor nanosystems. Semiconductors 47, 1626 (2013)

6. Pokutnyi, S.I., Gorbyk, P.P.: Quasi-zero-dimensional nanostructures: excitonic quasimolecules. J. Appl. Chem. 2, 1 (2014)

7. Frish, S.E.: Optical spectra of atoms. Nauka, Moscow (1963). [in Russian]

8. Bondar, N.V., Brodyn, M.S.: Spectroscopy of semiconductor quantum dots. Phys. E. 42, 1549 (2010)

9. Keldysh, L.V.: Coulomb interaction between electrons in thin films of semiconductors and semimetals. JETP Lett. 29, 621 (1979)

10. Pokutnyi, S.I., Gorbyk, A.P.: Local electron states in ellipsoidal nanosystems in homogeneous magnetic field. J. Nanostruct. Chem. 4, 100 (2014) 